\title{
Recent Numerical Advances in Fluid Mechanics
}

\section{Omer San}

School of Mechanical and Aerospace Engineering, Oklahoma State University, Stillwater, OK 74078, USA; osan@okstate.edu; Tel.: +1-405-744-2457; Fax: +1-405-744-7873

Received: 7 May 2020; Accepted: 11 May 2020; Published: 15 May 2020

In recent decades, the field of computational fluid dynamics has made significant advances in enabling advanced computing architectures to understand many phenomena in biological, geophysical, and engineering fluid flows. Almost all research areas in fluids use numerical methods at various complexities: from molecular to continuum descriptions; from laminar to turbulent regimes; from low-speed to hypersonic, from stencil-based computations to meshless approaches; from local basis functions to global expansions, as well as from 1st-order approximation to high order and spectral accuracy. Many successful efforts have been put forth in dynamic adaptation strategies, e.g., adaptive mesh refinement and multiresolution representation approaches. Furthermore, with recent advances in artificial intelligence and heterogeneous computing, broader fluids community has gained momentum to revisit and investigate such practices. This Special Issue, containing a collection of 13 papers, brings together researchers to address recent numerical advances in fluid mechanics.

Murea [1] presented an efficient semi-implicit monolithic method to simulate fluid-structure interaction problems within the Arbitrary Lagrangian Eulerian framework. The paper by Mohebbi et al. [2] exploits an accurate implementation of Kutta conditions for the airfoils with both finite angle and cusp trailing edges. Iqbal et al. [3] focused on the non-linear Schrödinger equation, and proposed a numerical method utilizing cubic B-spline Galerkin method. The paper by Karakouzian et al. [4] is devoted to the numerical modeling of hydraulic fracturing process in pressure tunnels. The authors performed a systematic finite element analysis to gauge guidelines for reducing the possibility of hydraulic fracturing in hydroelectric power plants. Cremades Rey et al. [5] concentrated their attention to the uncertainty quantification of Reynolds-averaged Navier-Stokes models using the open source package OpenFOAM. In their manuscript, Patel and Mathew [6] introduced a concept of adaptive explicit filtering for implicit large eddy simulations. The core idea is to utilize a second order accurate explicit filter in the presence of a discontinuity, and then gradually increase the order of explicit filter at the grid locations away from this discontinuity. The paper by Butcher and Spencer [7] takes up the concept of proper orthogonal decomposition (POD) for the identification of coherent structures. A new metric has been introduced for the selection of POD modes in reconstructing coherent or incoherent features. Idrissi et al. [8] dealt with an atmospheric dispersion study in highly realistic urban areas considering high level of details. They utilized large eddy simulations to explore the behaviour of the released pollutants in various topological settings. The article by Guilizzoni et al. [9] investigates the impact of multiple synchronized drops into a deep pool. In their paper, Bourantas et al. [10] put forth a strong form meshless point collocation method for solving the unsteady incompressible Navier-Stokes equations. Fakhari [11] considered to develop a wall model for large eddy simulations in simulating either the body fitted or immersed boundary problems. The paper by Ahmed and San [12] discusses a partitioning approach to break the Kolmogorov barrier in projection based reduced order modeling of transient flows. Finally, Xie et al. [13] introduced a machine learning approach to model the interaction between the resolved and unresolved modes in reduced order models.

Conflicts of Interest: The author declare no conflict of interest. 


\section{References}

1. Murea, C.M. Three-Dimensional Simulation of Fluid-Structure Interaction Problems Using Monolithic Semi-Implicit Algorithm. Fluids 2019, 4, 94. [CrossRef]

2. Mohebbi, F.; Evans, B.; Sellier, M. On the Kutta Condition in Compressible Flow over Isolated Airfoils. Fluids 2019, 4, 102. [CrossRef]

3. Iqbal, A.; Abd Hamid, N.N.; Ismail, M.; Izani, A. Soliton Solution of Schrödinger Equation Using Cubic B-Spline Galerkin Method. Fluids 2019, 4, 108. [CrossRef]

4. Karakouzian, M.; Nazari-Sharabian, M.; Karami, M. Effect of Overburden Height on Hydraulic Fracturing of Concrete-Lined Pressure Tunnels Excavated in Intact Rock: A Numerical Study. Fluids 2019, 4, 112. [CrossRef]

5. Cremades Rey, L.F.; Hinz, D.F.; Abkar, M. Reynolds Stress Perturbation for Epistemic Uncertainty Quantification of RANS Models Implemented in OpenFOAM. Fluids 2019, 4, 113. [CrossRef]

6. Patel, S.K.; Mathew, J. Shock Capturing in Large Eddy Simulations by Adaptive Filtering. Fluids 2019, 4, 132. [CrossRef]

7. Butcher, D.; Spencer, A. Cross-Correlation of POD Spatial Modes for the Separation of Stochastic Turbulence and Coherent Structures. Fluids 2019, 4, 134. [CrossRef]

8. Idrissi, M.S.; Ben Salah, N.; Chrigui, M. Numerical Modelling of Air Pollutant Dispersion in Complex Urban Areas: Investigation of City Parts from Downtowns Hanover and Frankfurt. Fluids 2019, 4, 137. [CrossRef]

9. Guilizzoni, M.; Santini, M.; Fest-Santini, S. Synchronized Multiple Drop Impacts into a Deep Pool. Fluids 2019, 4, 141. [CrossRef]

10. Bourantas, G.C.; Zwick, B.F.; Joldes, G.R.; Loukopoulos, V.C.; Tavner, A.C.; Wittek, A.; Miller, K. An Explicit Meshless Point Collocation Solver for Incompressible Navier-Stokes Equations. Fluids 2019, 4, 164. [CrossRef]

11. Fakhari, A. A New Wall Model for Large Eddy Simulation of Separated Flows. Fluids 2019, 4, 197. [CrossRef]

12. Ahmed, S.E.; San, O. Breaking the Kolmogorov Barrier in Model Reduction of Fluid Flows. Fluids 2020, 5, 26. [CrossRef]

13. Xie, X.; Webster, C.; Iliescu, T. Closure Learning for Nonlinear Model Reduction Using Deep Residual Neural Network. Fluids 2020, 5, 39. [CrossRef] 